\title{
LA RELATION ENTRE L'ÉTAT ET LA DETTE PUBLIQUE: LES APPROCHES GÉNÉRALES, LES LOGIQUES POLITIQUES, LA CRISE ET LE CAS ITALIEN ('70-'90)
}

\author{
LEONIDA TEDOLDI (*) \\ Nota presentata dal m.e. Silvio Beretta
}

(Adunanza del 26 ottobre 2017)

SUNTO. - Questo articolo mira ad analizzare, e a ripensare, le cause storiche, politiche e istituzionali della rapida crescita del debito pubblico negli anni settanta-ottanta e della sua strumentalizzazione condotta dai governi della cosiddetta "Prima Repubblica". Lo Stato italiano ha spesso vissuto al di sopra dei propri limiti e il rafforzamento del debito ha quindi funzionato come fattore indispensabile per la crescita economica. Se tale situazione ha portato inizialmente ad un consolidamento del rapporto tra lo Stato e i creditori - e quindi prevalentemente la società italiana - il protrarsi della crescita del debito in dosi massicce ha condotto progressivamente alla crisi complessiva del paese e quindi anche a quella di legittimazione istituzionale, che peraltro ancora oggi lo affligge.

$* * *$

ABSTRACT. - This article rethinks the political and institutional causes of the rapid debt growth and its exploitation in the italian "blocked" political system (so-called "First Republic"). Italian State has always lived above its means, with a constant imbalance between income and expenditure and at the same time expanding its distance with respect to society (but the debt was paid by social groups that took advantage of it). This process triggered off a perennial crisis of representation and strengthened the instability of relations between political institutions and society. Therefore, sovereign debt

(*) Dipartimento di Lettere Filosofia e Comunicazione, Università degli Studi di Bergamo, Italy. E-mail: leonida.tedoldi@unibg.it 
downturns are always crises of institutional legitimization and require a redefinition of the ways in which sovereignty and power are exercised. Thus, the article investigates the impact of the "political use" of the public debt by governments on the relationship between the State and society.

1.

La crise européenne des dettes souveraines a récemment mis sur le devant de la scène politique et médiatique la question de la crédibilité des États face aux marchés financiers. Les moments de crise sont l'occasion de s'interroger sur le processus historique de construction de la dette de l'État et sur ses liens avec l'enracinement des démocraties parlementaires.

Dans l'histoire occidentale, la dette publique a toujours été un facteur central dans le maintien et dans le renforcement du pouvoir politique, une sovereign decision déterminante. ${ }^{1}$ À la manière, plus générale, des décisions concernant la finance publique, elle fut souvent mise en œuvre par l'action politique des institutions, et beaucoup moins en suivant des théories axées sur la macroéconomique.

Pour cette raison, parler de dette publique signifie parler, avant même de politique économique, d'interactions et d'enchevêtrement entre les institutions, les forces et les mandataires sociaux organisés, les partis, les groupes de pression et les comportements des administrations publiques.

De plus, la gestion de la dette possède la particularité de placer les intérêts privés au centre de la question du soutien économique de l'État, créant par là un lien d'interdépendance forte entre le pouvoir politique et les citoyens créanciers - "le peuple de l'État" - lien complexe qui concerne notamment les relations de "confiance", de consensus $;^{2}$ du reste. Comme le suggère la recherche historique, les

1 K. Dyson, States, Debt and Power. 'Saints' and 'Sinners' in European History and Integration, Oxford, Oxford University Press, 2014 et R.W Kolb, Sovereign Debt. Theory, Defaults, and Sanctions, in Id. (ed.), Sovereign Debt from Safety to Default, Hoboken NJ, Wiley \& Sons, 2011, 3-13, H. Thompson, Debt and Power: The United States' Debt, Historical Perspective in International Relations, vol. 21 (3), 2007, 305-323 et aussi A. Downs, An Economic Theory of Democracy, New York, Harper, 1951.

2 L'historiographie anglo-saxonne parle également de good and bad debt. "The first narrative celebrates the developmental and entrepreneurial rolo of the State as an engine of innovation... The second narrative represented the State as à source of crony capitalism, which negates the vitality of free and open markets, ... 
crises de la dette sont toujours des crises de légitimation institutionnelle, et elles requièrent une redéfinition des manières dont la souveraineté est exercée.

Cependant, d'un autre point de vue, il convient d'ajouter que la Constitution italienne avait dessiné un régime parlementaire qui disposait d'une part d'une faible relation entre l'État et le marché, et d'autre part d'une division des pouvoirs au caractère précaire, sans sous-estimer d'autres questions importantes telles que l'absence de constitutionnalisation des partis.

Le but n'est donc pas tant d'analyser la gestion la gestion politique de la dette que de proposer une "analyse" complexe du phénomène de l'énorme croissance de la dette, et de son utilisation politique, pour comprendre les relations entre les institutions de l'État et les gouvernements ainsi que les relations entre les "citoyens-créanciers" et les acteurs institutionnels (en acceptant de prêter à l'État, les particuliers reconnaissent sa légitimité).

Les opérations de lancement des emprunts publics sont conçues comme des "plébiscites financiers" qui doivent servir de test à la légitimité du régime et des gouvernants. ${ }^{3}$

Dans les moments de crise, les États sont tentés de faire appel directement au public, notamment lorsque la défiance sévit sur les marchés financiers.

La dette publique est toujours une décision souveraine, qui implique le gouvernement et le parlement; ${ }^{4}$ en Italie "l'utilisation politique" de la dette publique a renforcé la légitimation du système politique et de son consentement enracinée dans la classe moyenne. En effet, l'action de recours à la dette exerce toujours une attraction à la fois pour les gouvernements et pour la société; ça crée l'illusion d'être en mesure de maintenir le développement et la croissance économique.'

and as the root cause of dependency culture, subordinating policies to the interests ... of recipients of State benefits" (Dyson, States, Debt and Power, cit., 3).

3 Voir N. Delalande, Les batailles de l'impôt. Consentement et résistances de 1789 à nos jours, Paris, Seuil, 2013 et aussi M. Lutfalla, Généralités sur la dette publique, en Id. (dir.), Une bistoire de la dette publique en France, Paris, Classiques Garnier, 2019, 28-37.

4 Voir V. Grilli D. Masciandro, G. Tabellini, Political and Monetary Institutions and Public Policies in the Industrial Countries, Economic Policy, 6 (13), 1991, 342-392.

5 Selon Thomas Piketty le recours à la dette publique favorise une 
Alors, à mon avis, la dette ce n'est qu'un "prétexte" pour parler de l'action de l'Exécutif, de la complexité des structures de l'État et des rapports entre les institutions politiques et la société. Qui contrôle réellement l'État et les politiques qu'il met en œuvre et après comment le contrôle de la croissance de la dette publique a affecté la structure des pouvoirs publics? ("La logique d'endettement contrôlé"). La question du contrôle de la dette est une partie intégrante de la stratégie de l'action politique, en particulier à partir de la seconde moitié des années soixante-dix et quatre-vingt. Et alors, est que la dette est l'âme du système ou sa déviation?

La dette publique constitue donc un bon observatoire des relations complexes et alors, peut constituer "une remarquable clef de lecture" a écrit Maurice Aymard; en outre Jean Yves Grenier soulignait que "la dette publique doit être conçu comme un système financier dont la gestion est négociée entre l'État, les corps intermédiaire, les financiers et les groupes sociaux les plus influents". ${ }^{6}$ Mais surtout les résultats de ce compromis dépendent du rapport de force de moment.

Selon la théorie économique, l'augmentation de la dette publique dans l' années soixante-dix est dû à un excès de la démocratie, tandis que selon le sociologue, Wolfgang Streeck, elle doit être liée à la transformation néo-capitaliste ou à l'involution du "capitalisme démocratique", qui a conduit à la crise de l' "État fiscal".

L'Etat débiteur peux-t-il, par conséquent, conduire à la désintégration de l'État? ou la dette et l'endettement c'est l'élément habituel du système capitaliste? A vraie dire, la dette publique est l'une des sources qui alimentent le cycle de la disponibilité de l'argent-consommation-production.

Pour cette raison je crois que la recherche historique devrait viser à analyser surtout la dette publique, son utilisation politique,

redistribution à l'envers: des plus pauvres à ceux qui ont les moyens de prêter de l'argent à l'État (qui devraient plutôt payer des impôts).

6 M. Aymard, En guise de conclusion, 473 et aussi J.Y. Grenier, Introduction. La dette d'État et la dette publique, en L. Quennonuëlle-Corre, Dette Publique et marchés de capitaux au XXe siècle: les poids de l'État dans le système financier français, en J. Andreau, G. Béaur, J.-Y. Grenier (dir.), La dette publique dans l'Histoire, Paris, Comité pour l'histoire économique et financière de la France, 2006, pp. 1-19. 
comme outil pour renforcer l'action de gouvernement, du consensus et le maintien de l'État dans son ensemble et pour mettre l'accent sur les mécanismes et les distorsions de la gestion politique et institutionnelle, afin de mieux comprendre la dynamique de la gouvernance au sein de l'État.

Par conséquent, la recherche devrait concentrer l'attention sur l'utilisation politique de la dette publique; le recours à l'endettement c'est aussi un dispositif politique, pour citer Foucault; c'est à dire l'utilisation politique de la dette par les gouvernements renforce la pression politique, l'action politique, par exemple, du Premier ministre, du Président du Conseil, (la crise du pouvoir exécutif, commencé il y a quarante ans avec la fin du keynésianisme, avant le choc pétrolier, et ça n'est pas encore conclue ${ }^{7}$ sur le parlement et l'administration centrale.

Dans les années soixante-dix, la transformation sociale, à la suite d'une défaillance de la structure de l'État et des grandes transformations du capitalisme, a conduit à l'augmentation de la dette publique, et cette augmentation a alimenté une crise dans le système institutionnel italien basé sur le concept que la dette publique était un véritable régulateur de l'économie et du consensus (pour vingt ans). Plus en générale, on peut dire que les démocraties occidentales étaient bien conscientes que la dette crée une relation avec les créanciers très complexes, qui concernent principalement la relation de "confiance", le consentement et la poursuite de l'imagerie sociale et symbolique. En même temps, les gouvernements savaient que, pour soutenir un État démocratique, avec une dette élevée, il était nécessaire satisfaire leurs créanciers. ${ }^{8}$ Mais également la dette publique peut devenir le "acheteur de la démocratie" en faveur d'une group étroit de créanciers, lorsque la dette publique est insoutenable.

7 Dans un contexte mondial, où les nouvelles orientations de la politique monétaire internationale ont conduit à une forte augmentation des taux d'intérêt, l'épuisement évident du paradigme fordiste-keynésien a conduit à la croissance de la pensé monétariste, qui a répondu à la volonté à surmonter les vieilles idées interventionnistes, qui n'avaient pas réussi à lutter contre la stagflation. Voir S. Cassese, Governare gli italiani. Storia dello Stato, Bologna, Il Mulino, 2013.

8 Voir W. Streeck, Buying Time: The Delayed Crisis of Democratic Capitalism, London-New York, Verso Books, 2014, 98-100. En Italie, la dette privée n'a jamais été au niveau des États-Unis, par exemple. 


\section{LE CAS ITALIEN}

L'analyse porte donc sur mes investigations concernant la politique, la policy, de la dette publique, au-delà des aspects économiques plutôt évidents, en tant que partie structurelle - et structurante - des institutions politiques. Ainsi, l'étude de la forte croissance de la dette publique italienne à partir des années 1970 devient un "prétexte" utile, ou un sujet, pour repenser en profondeur les mécanismes, la construction et le maintien de l'État dans son ensemble, mais également les relations entre le gouvernement, le parlement et les magistratures de contrôle telles que la Cour des Comptes.

Elle permet en même temps de revoir certains lieux communs, comme celui qui attribue essentiellement la croissance de la dette italienne aux besoins de développement de l'État providence ou des dimensions de l'État en général.

En Italie, le passage entre la crise budgétaire de l'État et la construction de l' "État débiteur" a commencé vers la fin des années soixante-dix, dans un contexte constitutionnel des relation parmi les pouvoir publiques problématique pas solide. ${ }^{9}$

À la fin des années soixante-dix, l'Italie fait face à la deuxième vague de la récession, en raison de la crise du pétrole, avec ses inefficacités déjà manifestes, d'une part, et avec la puissance écrasante de son appareil administratif de l'autre, qui portait les problèmes graves des déficits budgétaires et structurelles, qui avait suscité l'inflation. Il ne faut pas oublier le rôle prépondérant de l'État dans le processus de modernisation et de libéralisation, comme en France, Allemagne et Japon, tandis que les pays anglo-saxons, États-Unis, Grande Bretagne et Canada, avaient déjà effectué la mutation de leur système financier dans les années '70.

En ce moment, d'une part, la croissance de l'inflation a permis à l'État d'emprunter à des coûts réels décroissants et parfois négatives (parce que la dette a été payée à des groupes sociaux qui ont profité), tandis que l'autre l'action politique a renforcé l'idée de la croissance au

9 Voir G. De Luca, Debito pubblico, mercato finanziario ed economia reale nel Ducato di Milano e nella Repubblica di Venezia tra XVI e XVII secolo, in Id., A. Moioli (a cura di), Debito pubblico e mercati finanziari in Italia secoli XIII-XX, Milano, FrancoAngeli, 2007, 119-146 et Delalande, Les batailles de l'impôt, cit. 
détriment de l'avenir, dans la conviction que l'État aurait toujours le plein contrôle sur la monnaie et l'économie réelle. ${ }^{10}$

L'Italie a toujours été caractérisée par des niveaux élevés de dette publique, pendant une partie considérable de son histoire unitaire. En effet, le rapport entre la dette et le PIB a été de plus de 100\% pendant 63 ans, dépassant $60 \%$ pendant 111 ans. Ce n'est pas sans raison que les périodes de forte réduction de la dette sont toutes condensées dans la phase de ce que l'on a appelé le "miracle économique", à savoir en pleine croissance économique. ${ }^{11}$

Donc, à partir de la fin des années 1970, le passage vers l' "État débiteur" - du capitalisme de l'épargne à celui de la dette, aurait dit Ralf Darhendorf - se mêla étroitement à la croissance persistante de l'inflation, laquelle a permis en un certain sens à l'État de s'endetter à des coûts réels décroissants et parfois négatifs, alors que sur un autre versant, il était mêlé au processus de détérioration de la centralité du parlement au profit du renforcement progressif du rôle du gouvernement dans la formation du budget public, et donc dans la politique de la dépense.

Ce processus était également favorisé par la conviction politique que l'État aurait toujours le plein contrôle sur la dette, sur la monnaie et sur l'économie réelle, et il donnait l' "illusion rationnelle", presque un réflexe adaptatif, d'une croissance avec des coûts sociaux réduits, à la charge de l'avenir. ${ }^{12}$

Entre 1976 et 1979, et surtout après la loi financière de $1978,{ }^{13}$ la

10 L. Raffaelli, La fabbrica del disavanzo: la crisi fiscale dello Stato italiano, Milano, De Donato, 1982 et N. Ferguson, C.S. Maier, E. Manela e D.J. Sargent (eds.), The Shock of the Global. The 1970s in Perspective, Cambridge MA, Harvard University Press, 2010.

11 F. Balassone, M. Francese, A. PACE, Debito pubblico e crescita economica, in G. Toniolo (a cura di), L'Italia e l'economia mondiale dall'Unità ad oggi, Venezia, Marsilio, 2013, 711-734.

12 Ce point de vue était fonctionnelle dans le processus politique généré par le "compromis historique" et par la saison de la "solidarité nationale", qui, en termes économiques, se tient sur l' "échange" entre la défense et la maîtrise des coûts de maind'œuvre, d'une part, et l'augmentation de l'investissement public et l'expansion des garanties des droits sociaux, de l'autre.

13 Jusqu'à la loi 468 de 1978, qui institua la loi "financière", la politique de l'endettement découlait surtout de normes d'autorité qui régissaient la contraction d'emprunts spécifiques, imposant au budget de transposer les décisions d'endettement précédentes. Voir aussi Senato della Repubblica, VIII Legislatura, Decisione e relazione 
stratégie - destinée à durer - du recours à la dette pour financer les dépenses courantes s'est consolidée. ${ }^{14} \mathrm{Au}$ début, ce fut grâce à un arrêt de la Cour constitutionnelle qui avait reconnu, en contournant l'art. 81 Cos. ${ }^{15}$ que le recours à l'émission d'emprunts était admissible dans le cas où la couverture financière des lois se démontrerait "suffisamment sûre". Ce fut ensuite par l'affinement d'une tactique imposée par les gouvernements, qui engageait des parts de fonds ("globales", reliquats budgétaires, etc..), chaque année plus élevées, pour le soutien financier de certaines lois sur les dépenses - sans les "renégocier" annuellement avec le parlement - et donc souvent dépourvues d'une réelle couverture tout de suite après la première année. Ce fut le prétexte avancé par l'Exécutif pour recourir à l'emprunt. ${ }^{16}$

Dès lors, la dette publique se transforma en politique de dépense et de croissance, et elle devait garantir aux gouvernements suivants la stabilité d'un système institutionnel fragile. Dans le même temps, l'Exécutif pouvait s'octroyer des espaces d'action par rapport à la centralité du parlement, en amorçant un processus d'autolégitimation servant de point de résolution d'une "médiation politique continue", qui préservait la solidité des relations institutionnelles entre les ministères. Dès 1978, les dépenses courantes dues à l'accroissement des "dimensions" de l'État avaient atteint un taux d'incidence $(33,9 \%)$ plus haut que jamais sur le montant des dépenses globales.

Dans de telles circonstances, alors que par ailleurs la part de la dépense publique non couverte par des recettes dépassait $55 \%$, et que la croissance constante du déficit grimpait de façon inexorable, la dette publique se dilatait inévitablement, pour atteindre environ $26 \%$ par an, dépassant largement la limite de $60 \%$ sur le PIB. ${ }^{17}$

Dans les années soixante-dix et le début de la prochaine décennie,

della Corte dei Conti sul rendiconto generale dello Stato per l' esercizio finanziario, 1978.

14 D. Franco, L'espansione della spesa pubblica in Italia, Bologna, Il Mulino, 1993.

15 "Avec la loi d'approbation des comptes, il est impossible de créer de nouveaux impôts et de nouvelles dépenses. Toute autre loi important de nouvelles dépenses ou des dépenses plus importantes doit indiquer les moyens d'y faire face”.

16 Cette situation devint évidente, par exemple, avec l'intervention du ministre Gaetano Stammati face à l'augmentation dangereuse de l'endettement des communes en 1977.

17 Voir B. Andreatta, F.M. Pandolfi, Relazione sulla situazione economica del paese (1979), Roma, Istituto Poligrafico dello Stato, 1980. 
bien que l'Italie était encore en pleine expansion de l'élan cyclique de l'investissement, la longue période de lien entre l'entreprise et l'intervention publique a été réduite et aussi le rôle de la politique économique et sociale dans la poursuite du développement du pays. ${ }^{18}$ Dans le même temps, les politiques de dépenses sociales avaient déjà défini la stratification sociale.

En outre, la croissance de la dette publique et son utilisation politique ont façonné les institutions, au moins la transition entre la soidisant "parlementarisme intégral" (hégémonie du Parlement sur le gouvernement) et le renforcement du rôle du gouvernement (seulement politique et non constitutionnel), qui se déroule entre la fin des années soixante-dix et la décennie suivante.

Le système politique et institutionnel allait traverser une période de profonde incertitude, (également caractérisé par une forte concurrence entre les chrétiens-démocrates et le Parti socialiste), qui avait réduit la primauté du politique. ${ }^{19}$

En fait, une période de changement profond se consolidait. L'épuisement de l'expérience de la grande réunion entre DC (Démocratie Chrétienne) et PCI (Parti Communiste) avait accéléré la détérioration de la légitimité des institutions, qui ne étaient pas en mesure de gérer la gouvernance publique de l'économie, dans le visage d'une saison exceptionnelle de la hausse des dépenses publiques, comme celle de '79 -'80, qui avait signalé des effets lourds sur le déficit de l'État. ${ }^{20}$

Ce sont les années, en somme, où les racines sociales des classes moyennes se sont consolider et développer sous la pression d'un haut niveau constant de dépenses sociales.

Aux début des années 1980, les limites politiques et administratives de contrôlabilité de la dette étaient déjà évidentes. Elles entraînè-

18 Voir D. Fausto, La politica del debito pubblico dell'Italia repubblicana nelle Relazioni della Banca d'Italia, in De Luca, Moioli (a cura di), Debito pubblico e mercati finanziari in Italia, cit., 685.

19 Voir Censis, XIV rapporto/1980 sulla situazione sociale del paese, Roma, Censis, 1980, 93 et R. Romanelli (a cura di), Storia dello Stato italiano. Dall'Unità ad oggi, Roma, Donzelli, 1997 et aussi L. Tedoldi, Storia dello Stato italiano. Dall'Unità al XXI secolo, Roma-Bari, Laterza, 2018.

20 Voir Censis, XVII rapporto/1983 sulla situazione sociale del paese, Roma, Censis, 1983, 12 et après voir l'intervention parlementaire de B. Kessler, Camera dei Deputati, Atti parlamentari, VIII Legislatura, seduta del 30 marzo 1982, 43117. 
rent un conflit entre l'Exécutif et d'autres organes de l'État, (Cour des comptes et Comptabilité générale) et la définition d'une ligne de collision avec les Régions, qui venaient d'être instituées, à un moment où le Trésor public, surtout après les "divorce" avec la Banque d'Italie de 1981, gérait une partie de la comptabilité générale dans un contexte de fort contraste entre les centres de dépense ministériels.

En conséquence, même si le "divorce" avait relancé le rôle névralgique des magistratures techniques indépendantes en fonction de contrepoids aux organes du pouvoir politique, ainsi que le rôle institutionnel du parlement et du gouvernement en matière économique, le processus décisionnaire d'approche budgétaire restait souvent immergé dans une "incertitude stratégique" et dans un "consociativisme compétitif" entre la majorité et l'opposition communiste. Cette condition a favorisé le "parti de la dépense" (qui s'alimentait également des conflits au sein de la DC), mettant en évidence la faiblesse des gouvernements face à la nécessaire durabilité économique du renforcement de l'État en général. ${ }^{21}$

Dans tous les cas, dès la fin de l'année 1981, la fonction de couverture de la dette commençait à être assumée majoritairement par la dette "fluctuante", alimentant la croissance rapide des intérêts sur les BOT (Bons "ordinaires" du Trésor).

En substance, au cours des années 1980, l'État débiteur italien a bien conduit à la désagrégation de l'État fiscal et à la dégénération (en partie seulement, je crois) de la démocratie, comme le soutient au niveau européen Wolfgang Streeck, ${ }^{22}$ mais il n'en reste pas moins qu'il a également débouché, en Italie, sur le développement d'une relation significative avec les classes moyennes: partie décisive du "peuple de l'État", qui envahit également les structures étatiques, ce qui crée des zones importantes et stratégiques de privilège et surtout de rente solide.

Il aura fallu de six à sept ans pour que se crée le "socle dur" de la dette publique italienne, avec un bon d'environ $40 \%$ qui a donné un rapport de $90 \%$ avec le PIB, à la fin de la décennie.

Il semble évident que pendant la saison des gouvernements

21 Voir G. Brosio, C. Marchese, Il Potere di spendere. Economia e storia della spesa pubblica dall'Unificazione ad oggi, Bologna, Il Mulino, 1986 et V. Tanzi, L. Schuknecht, Public Spending in the 20th Century. A Global Perspective, Cambridge (UK), Cambridge University Press, 2000.

22 W. Streeck, Die Vertagte Krise des demokratischen Kapitalismus, Berlin, Suhrkamp Verlag, 2013. 
"laïcs" (Spadolini, Craxi) le processus de verticalisation et de délimitation de l'aire décisionnaire de l'Exécutif à un nombre restreint d'acteurs institutionnels a débouché sur une utilisation politique bien précise de la dette publique, dans le but de renforcer la légitimation constante du gouvernement - qui s'était alors transformé en lieu de conflits permanents entre les partis, avec pour effet d'élargir définitivement la capacité décisionnaire de l'Exécutif, en tant qu'acteur normatif autonome. ${ }^{23}$

Il ne fait aucun doute que le choix du maintien de l'endettement à des niveaux fort élevés, inconnus aux autres démocraties européennes, était également déterminé par la pression des intérêts dans la société. Ceux-ci étaient contraires à tout redimensionnement de la politique de la dette comme stratégie de croissance, et surtout de la politique "consociative", qui générait un flux constant de richesses entre les mains des détenteurs de la dette du fait qu'à l'époque, les "familles" détentrices de titres d'État représentaient encore près de $56 \%$ du total des acquéreurs. L'action politique avait augmenté les dépenses publiques - ce qui se passait dans sa troisième phase d'expansion (1978 à 1985) - à la recherche d'un consensus "dans les limites de gouvernabilité", selon certains commentateurs, provoquant un problème de légitimité, destiné à augmenter. C'est-à-dire, l'incapacité des gouvernements de forcer les exigences sociales au sein des ressources disponibles dans un contrôle fiscal, tel que Michele Salvati a revendiqué à plusieurs reprises. ${ }^{24}$

Les échecs successifs des "politiques de réduction de la dette publique" de la fin des années 1980 étaient enlisés dans les contradictions entre les ambitions des Exécutifs renforcés, tant au niveau structurel que dans leur style décisionnaire, ainsi que dans un système général d'interactions entre le gouvernement, le parlement et les partis, qui était en train de se relâcher. ${ }^{25}$

23 L. Verzichelli, Le politiche di bilancio, Bologna, Il Mulino, 1999 et L. Tedoldi, Il conto degli errori. Stato e debito pubblico in Italia, Roma-Bari, Laterza, 2015, $72-85$

24 M. Salvati, Occasioni mancate. Economia e politica in Italia dagli anni '60 a oggi, Roma-Bari, Laterza, 2000, 55-57 et aussi Senato della Repubblica, Camera dei Deputati, IX Legislatura, Relazione della Corte dei Conti sul rendiconto generale dello Stato per l'esercizio finanziario, 1984.

25 Dans les années quatre-vingt commence la séparation entre le gouvernement et l'administration centrale d'une part et les Régions de l'autre part et la transformation de l'État régulateur de l'économie, comme dans d'autres pays. 
Quoi qu'il en soit, le coût de la dette correspondait désormais à celui des transferts de l'État aux collectivités locales, aux organismes de prévoyance et aux services de santé.

Au bout de 7 à 8 ans, le "divorce" entre le Trésor public et la Banque d'Italie (1981) n'avait pas encore favorisé de politiques de stabilisation et de rééquilibre de la finance publique; au contraire, il avait surtout confirmé une clairvoyance déficiente et une crise de crédibilité des gouvernements et de la classe politique face à l'autonomie de la Banque centrale. ${ }^{26}$

Dans tous les cas, cette politique de la dette mettait tous les partis d'accord (y compris les syndicats), conscients qu'ils étaient des dynamiques à risque de cette stratégie, mais incapables de sortir du labyrinthe du système politique italien "gelé", toujours "prisonnier" d'une situation embrouillée et désormais consolidée: l'endettement fluctuant à des taux élevés fournissait un soutien à l'épargne pour les classes moyennes, et à son tour, une partie de cette épargne était investie en nouvelle dette publique sous une forme plus large, créant une vaste base de consensus. En outre, l'utilisation politique (100\% du PIB vers la fin des années Quatre-vingt) de la dette publique a eu une forte influence sur le maintien des liens institutionnels entre l'Exécutif et les ministères. Et le Trésor gère une partie de la comptabilité dans un contexte de forte conflit parmi les centres de coûts. ${ }^{27}$ Mais, il y a des limites de contrôlabilité de la dette. ${ }^{28}$ Les problèmes politiques et institutionnels sur la gestion des comptes et des dépenses publiques. Le renforcement de la centralité du gouvernement dans la formation de budget public devient un élément de conflit entre les Pouvoirs publics, à commencer par la relation entre le gouvernement, le Parlement, la Cour des comptes et le Bureau de la Comptabilité générale.

Quoi qu'il en soit, d'un côté la dette publique continuait d'augmenter, en même temps que les besoins de l'État et la dépense publique (les recettes restaient le vrai problème, avec le phénomène macroscopique de la fraude fiscale), et d'autre part, la perte progressive de

26 M.L. Marinelli, L'indipendenza della Banca d'Italia dal governo negli anni Ottanta: cause interne e internazionali, Studi e note di economia, XVI, 2, (2011), 133-170.

27 Voir S. Cassese, Espansione e controllo della spesa pubblica, in Il Mulino, 3 , 1983, 371-372.

28 F. Giavazzi, L. Spaventa (eds.), High Public Debt: the Italian Experience, Cambridge (UK), Cambridge University Press, 1988. 
contrôle du gouvernement s'accentuait, malgré la loi sur le droit de la présidence du Conseil de 1988 qui imposait la redéfinition d'une relation étroite entre le président du Conseil, le ministre du Trésor public et celui du Budget.

Dans les années 1990, l'orientation des gouvernements "techniques" (à partir d'Amato) fut celle de la réduction de la dépense d'intérêts, de l'allongement de la vie de la dette et de la réduction de l'endettement net de l'État, qui était par ailleurs déjà en cours depuis 1990 (réduit d'un point de pourcentage en deux ans environ), imposant une orientation politique "régulatrice" par rapport à l'attitude "distributrice" de la décennie précédente.

Cette orientation post-Maastricht façonna la "phase de passage" vers la recherche de politiques de stabilité, dans un contexte où les transferts de ressources de l'État se limitaient à ses participations, et où l'Union monétaire allait dépouiller les gouvernements de leur autonomie.

La crise du système politique, le phénomène Tangentopoli et la réunification allemande entraînèrent une augmentation des taux des intérêts sur la dette. Cependant, je pense que les difficultés politiques de contrôle de la croissance de la dette n'engendrèrent pas la "dénaturation" de la capacité de l'État (ni celle des autorités monétaires, du Trésor public ou encore de la Banque d'Italie) à graduer les charges budgétaires par rapport aux exigences de développement économique et social. Toutefois, alors que les tentatives liées à la "phase de réduction" du déficit alimentaient une action politique de redistribution intra-générationnelle, la croissance de la dépense publique, elle, atteignait $58 \%$ du PIB en $1993 .{ }^{29}$

En conséquence, l'objectif du gouvernement de cette année-là, conduit par Carlo Azeglio Ciampi, ancien gouverneur de la Banque Centrale, ne pouvait être que la réduction de la dépense d'intérêts en même temps que la reconstruction opiniâtre de la confiance du "peuple de l'État"”, la crédibilité de la politique dans le pays et en Europe, et une série de changements dans l'organisation administrative, comme dans la relation entre le parlement et la Cour des comptes.

Il se peut donc, vers le milieu des années 1990 comme jamais, que

29 F. Balassone, M. Francese, A. Pace, Debito pubblico e crescita economica, in G. Toniolo, (a cura di), L'Italia e l'economia mondiale dall'Unità a oggi, Venezia, Marsilio, 2013, 711-734. 
la crise financière manifeste de l'État ait touché en profondeur les relations entre les institutions, mais surtout entre le gouvernement et le parlement. Bien entendu, cette situation avait une incidence déterminante sur les politiques de la dette du Trésor public ${ }^{30}$ et sur la viabilité des comptes publics au niveau européen.

En effet, la ratification du traité de Maastricht est intervenue de manière appuyée dans la policy financière de la dette, et a enclenché un processus de redéfinition très progressif des politiques de la dette (qui se résumèrent principalement à un effort de maitrise des dépenses). Cela signifiait, dans l'immédiat, la réduction de l'intervention publique dans certains secteurs de fourniture de services, et la recherche de l'efficacité.

L'entrée dans l'Union monétaire changea définitivement les stratégies du gouvernement et du Trésor public, qui s'orientèrent vers une politique de restriction des titres à taux variable, ou avec des échéances à court terme. Toutefois, cette entrée montra à la classe politique italienne les potentialités de l'augmentation très significative des acheteurs étrangers de la dette italienne.

Pendant la deuxième moitié des années 1990, dans les relations entre le gouvernement et le parlement, l'exécutif poussait à une action d'assainissement équilibrée, où prédominait l'attention à la gouvernance de la dépense, et de l'autre côté, le parlement imposait une plus grande intervention politique sur le prélèvement fiscal, supérieure à celle qui était souvent prévue dans la programmation du DPEF (Document de programmation économique).

Le gouvernement Prodi est, peut-être plus que d'autres, celui qui a affronté ou tenté d'affronter les questions cruciales portant sur les phases gouvernementales et administratives de la manœuvre budgétaire, intervenant sur le plan législatif au niveau de la transparence, de la complexité excessive et de la faible flexibilité des procédures de gestion de l'exercice financier.

En outre, la loi adoptée par ce gouvernement, qui imposait l'unification des ministères du Trésor public et du Budget, renforça le rôle du Trésor public en qualité de garant de l'équilibre financier.

C'est peut-être également à cause de cette pression politique et

30 Le Trésor public disposait jusqu'alors d'un “droit de découvert” jusqu'à $14 \%$ de la dépense budgétaire. 
institutionnelle que l'exécutif parvint à construire une stratégie de maîtrise du besoin public, en contrôlant les flux de trésorerie de la balance commerciale de l'État. Pour ce faire, il réduisit les octrois de crédits de caisse sur les transferts aux collectivités ayant des soldes importants de trésorerie, ainsi qu'aux titulaires de comptes courants qui avaient une comptabilité particulière auprès de la Trésorerie de l'État (collectivités locales, universités et collectivités mineures). De plus, ne négligeons pas le fait que même après la réforme de l'IRAP en 1997, l'orientation générale de l'État continua à s'accentuer, consistant à maintenir le contrôle sur l'action des Régions dans le domaine de la santé, au moyen du levier financier.

La réduction du coût de la dette - déterminée par l'imposition de limites aux décaissements - limitait alors la dépense nette d'intérêts autour de $42 \%$ du produit (atteint en 1995), ce qui permettait une réduction de la dépense publique à 51,5\% par rapport au PIB et forçait l'endettement à rester en dessous de 120\%.11

En même temps - et en quatre à cinq ans seulement (1995-1999) -, la propriété de la dette se modifia de manière radicale et transforma la relation entre la société italienne et l'" État débiteur", fortement conditionnée par le climat des comptes dramatiques de l'économie, par la perte de références politiques et par la dégradation du tissu social après la crise de 1992. D'un autre point de vue, la modification des orientations stratégiques en Europe - avec la large majorité au sein du parlement italien permirent au gouvernement de mener à bien une action plus souple sur la dépense publique, laquelle connut une baisse constante de 1996 à 1999 et passa de plus de $11,5 \%$ à $6,5 \%$ du PIB annuel.

Les gouvernements qui ont suivi (D'Alema) avaient la particularité d'être des gouvernements exécutifs de transition, qui réussirent toutefois à mener à terme des privatisations dans le secteur de l'énergie et du transport. Parallèlement, on assista au développement de la croissance de la bourse et à un boom des marchés boursiers, également et

31 Dans les années quatre vingt-dix les gouvernements ont eu l'occasion de stabiliser le système et de renforcer le rôle de l'exécutif dans la formation de budget de l'État, mais ils ont échoué. Ils peuvent seulement réduire les dépenses des intérêts de la dette (le processus de privatisation des grandes structures industrielles de l'État avance, pas bien). Voir D. Fausto, La politica del debito pubblico dell'Italia repubblicana nelle Relazioni della Banca d'Italia, in De Luca, Moioli (a cura di), Debito pubblico e mercati finanziari, cit., 661-697. 
surtout à la suite du bond des investissements des petits épargnants, et cela n'a pas produit d'effets négatifs sur les comptes publics. La vente de la première tranche de la compagnie d'électricité ENEL eut un impact supplémentaire sur la réduction de la dette.

Dans le même temps, la réduction de la dépense d'intérêts se confirmait, et même si la dette tournait encore autour de niveaux doubles de la France et de l'Allemagne, elle est restée "longue", et donc gérable au niveau politique plus encore qu'au niveau économique.

La dette augmente et change sa composition et la majorité des créanciers sont les banques étrangères; le problème est la relation avec l'Union Européenne. Les créanciers privés ont un droit de veto sur les politiques publiques qui sont menées dans les pays endettés. Ils décident si les mesures sont suffisantes ou pas, et ils acceptent ou non une diminution de leur créance. En effet, l'incidence réduite de BOT et de CCT (Certificats de Crédit du Trésor) sur le total de la dette (de 59\% du total en 1990 à $29 \%$ en 1999) atténua la relation temporelle entre la variation des taux et les charges d'intérêts. De plus, entre 1996 et 1999 , les banques italiennes recommencèrent à acheter des titres, jusqu'à près de $40 \%$ du total (en 1991, elles en détenaient 25\%), mais surtout, les investisseurs étrangers passèrent de $15 \%$ en 1991 à $20 \%$ environ en 1996, à 38\% en 1999, constituant avec les établissements de crédit italiens près de $80 \%$ des détenteurs de titres de la dette italienne. ${ }^{32}$

L'augmentation des investisseurs étrangers fut certainement liée aux phases de la nouvelle saison politique et économique inaugurée avec l'unification de l'Europe post-Maastricht et avec les dynamiques des marchés financiers internationaux, mais elle traça une ligne de partage des eaux pour l'histoire des politiques de la dette publique en Italie, d'autant qu'elles entrèrent dans la liste des souscripteurs de certains pays européens de premier plan, non seulement l'Allemagne, mais aussi la France et le Royaume-Uni.

32 Voir R. Artoni, Il debito pubblico in Italia dall'unità ad oggi, in P. Ciocca, G. Toniolo, (a cura di), Storia economica d'Italia, Roma-Bari, Laterza, 2003, 269-380; M. Francese, A. Pace, Il debito pubblico italiano dall'Unità ad oggi. Una ricostruzione della serie storica, in Questioni di Economia e Finanza (Banca d'Italia), 2008, 31 et aussi P. De Ioanna, Parlamento e spesa pubblica, Bologna, Il Mulino, 1993 et Id. Debito pubblico e classe politica: uno sguardo d'insieme sulla Prima Repubblica, in S. Colarizi, A. Giovagnoli, P. Pombeni (a cura di), L'Italia contemporanea dagli anni Ottanta a oggi, III, Istituzioni e politica, Roma, Carocci, 2014, 141-158. 
Toutefois, cette tendance ne poussa pas les gouvernements italiens à miser sur les épargnants étrangers.

Aussi, à la fin des années 1990, lorsque l'État redevint un élément charnière dans la médiation des intérêts économiques (au détriment de son rôle de garant des droits du citoyen, et au beau milieu du développement d'une "privatisation" des relations citoyen/État et marché), la croissance de la dette publique connut une réduction et un ralentissement grâce à une série importante d'excédents primaires, obtenus avec des coupes dans les dépenses de santé, le gel des investissements publics, l'augmentation de la pression fiscale et les privatisations. Cependant, ces mesures n'ont pas constitué le fondement d'un nouveau départ qui aurait permis aux futurs gouvernements d'orienter la gouvernance de la dette au sein des "politiques européennes italiennes" destinées à encourager la stabilisation de l'endettement public. ${ }^{33}$ En effet, en 2001, peu de temps après la naissance de l'Euro, on assista à des rechutes de l'effort du processus d'accrochage à la monnaie unique ${ }^{34}$ qui rendirent manifeste l'incapacité italienne de donner corps à la requalification et à la réduction de la dépense courante, dans un contexte où le taux de croissance du PIB italien était au niveau le plus bas de tous les pays de l'Union européenne. ${ }^{35}$

33 F. Cavazzuti, La Repubblica in transizione (1989-94). Debito pubblico e fiscalità: le scelte politiche, in S. Colarizi, A. Giovagnoli, P. Pombeni, (a cura di), L'Italia contemporanea dagli anni Ottanta, cit., 159-184.

34 D. Velo, La neutralizzazione della moneta e del debito pubblico e la centralità della politica fiscale. Il modello europeo in divenire, in S. Beretta, F. Osculati (a cura di), Verso un debito pubblico europeo?, Soveria Mannelli, Rubbettino, 2012, 85-118.

35 Voir G. Amato, A. Graziosi, Grandi illusioni. Ragionando sull'Italia, Bologna, Il Mulino, 2013. 
